\title{
Heterogeneity in the in vitro susceptibility of Loa loa microfilariae to drugs commonly used in parasitological infections
}

Abdel J. Njouendou ${ }^{1 \dagger}$, Fanny F. Fombad ${ }^{1 \dagger}$, Maeghan O'Neill ${ }^{2}$, Denis Zofou ${ }^{3}$, Chuck Nutting ${ }^{4}$, Patrick C. Ndongmo ${ }^{1}$, Arnaud J. Kengne-Ouafo ${ }^{1}$, Timothy G. Geary ${ }^{2}$, Charles D. Mackenzie ${ }^{5,6+}$ and Samuel Wanji ${ }^{{ }^{* \dagger}}$ (D)

\begin{abstract}
Background: Co-infection with loiasis remains a potential problem in control programs targeting filarial infections. The effects of many anti-parasitic drugs often administered to Loa loa infected people are not well documented. This study compared the in vitro activity of several of these drugs on the viability of L. loa microfilariae (mf).

Methods: Human strain L. loa mf were isolated from baboon blood using iso-osmotic Percoll gradient, and cultured in RPMI 1640/10\% FBS with antimalarial drugs (mefloquine, amodiaquine, artesunate, chloroquine and quinine), anthelmintics (ivermectin, praziquantel, flubendazole and its reduced and hydrolyzed metabolites), two potential trypanocidal agents (fexinidazole and Scynexis-7158) and the anticancer drug imatinib. The drug concentrations used varied between $0.156 \mu \mathrm{g} / \mathrm{ml}$ and $10 \mu \mathrm{g} / \mathrm{ml}$. Mf motility ( $C R_{50}=50 \%$ immotility) and a metabolic viability assay (MTT) were used to assess the effects of these drugs on the parasites.

Results: Mf in control cultures showed only a slight reduction in motility after 5 days of culture. Active inhibition of Loa loa motility was seen with mefloquine and amodiaquine ( $C R_{50}$ values of 3.87 and $4.05 \mu \mathrm{g} /$ $\mathrm{ml}$, respectively), immobilizing $>90 \% \mathrm{mf}$ within the first 24 hours: mefloquine killed the $\mathrm{mf}$ after 24 hours of culture at concentrations $\geq 5 \mu \mathrm{g} / \mathrm{ml}$. SCYX-7158 also induced a concentration-dependent reduction in mf motility, with $>50 \%$ reduction in $\mathrm{mf}$ motility seen after 5 days at $10 \mu \mathrm{g} / \mathrm{ml}$. The anticancer drug imatinib reduced mf motility at 10 $\mu \mathrm{g} / \mathrm{ml}$ from the first day of incubation to $55 \%$ by day 5 , and the reduction in motility was concentration-dependent. Praziquantel and fexinidazole were inactive, and FLBZ and its metabolites, as well as ivermectin at concentrations $>5$ $\mu \mathrm{g} / \mathrm{ml}$, had very minimal effects on mf motility over the first 4 days of culture.
\end{abstract}

Conclusions: The considerable action of the anti-malarial drugs mefloquine and amodiaquine on Loa $\mathrm{mf}$ in vitro highlights the possibility of repurposing the existing anti-infectious agents for the development of drugs against loiasis. The heterogeneity in the activity of anti-parasitic agents on Loa loa $\mathrm{mf}$ supports the need for further investigation using animal models of loiasis.

Keywords: Antimalarial, Anthelmintics, Trypanocides, Imatinib, Loa loa microfilariae

\footnotetext{
* Correspondence: swanji@yahoo.fr; samwandji@gmail.com

${ }^{\dagger}$ Equal contributors

${ }^{1}$ Parasites and Vectors Biology Research Unit (PAVBRU), Department of

Microbiology and Parasitology, Faculty of Science, University of Buea, Buea,

Cameroon

Full list of author information is available at the end of the article
} 


\section{Background}

Loiasis, a neglected tropical disease also known as African eye worm, is caused by the nematode Loa loa, transmitted through the bite of deer flies (Chrysops spp.). Loiasis is thought to cause relatively little pathology except for localized mild to moderate pruritus and oedema, and distress due to the occasional sub-conjunctival migration of the adult worm. Although the disease is endemic in Central and West Africa [1], 101 cases were reported outside Africa in the past 25 years (1986-2011), of which 61 (60. $4 \%)$ were in Europe and 31 (30.7\%) in the USA [2]; however, all these patients had spent time in an African endemic area.

Although relatively asymptomatic when untreated, $L$. loa-infected people, when administered the anti-parasitic agents commonly used for other filarial infections such as onchocerciasis and lymphatic filariasis, may experience severe adverse effects, especially in people carrying very high loads of circulating $L$. loa $\mathrm{mf}$ (> $8000 \mathrm{mf} / \mathrm{ml}$ ) treated with diethylcarbamazine or ivermectin [3-5]. This posttreatment phenomenon has been an impediment to programmes administering ivermectin to eliminate onchocerciasis and lymphatic filariasis, both of which can co-exist with loiasis $[4,5]$. Fatal complications involving encephalopathy have been documented during the treatment of loiasis patients $[3,6,7]$. Further, the cross-reactivity of the Immuno-chromatographic card (ICT) with loiasis [8,9] has impaired the mapping of lymphatic filariasis in the rainforest block of central Africa, where loiasis is endemic.

No registered safe drug is available for the control of loiasis and no vaccine is available for loiasis or other filarial infections, despite the considerable efforts made to understand the mechanisms and major effectors of the immune response induced by the parasite in the host [10,11]. Pending the development of such a vaccine, the development of a drug that can safely clear the infection would be highly valuable for control and elimination programmes.

In the search for a safe drug for use in loiasis regions, there is a need of an in vitro system to study the effects of candidate drugs on the parasite viability. Although there has been considerable success in developing protocols to maintain these parasites in primates $[7,12$, 13], culture systems more amenable for higherthroughput drug testing remain unavailable. Baboons, along with mandrills, are important models for the study of $L$. loa since they, like humans, are susceptible to infection and develop prolonged microfilaraemia without developing clinical disease [7, 13]. Despite the success of maintaining $L$. loa parasites in baboons, this model is practically inappropriate for in vivo drug screening purposes at large scales; the numbers required and cost of animal maintenance, along with ethical and environmental constraints, prevent the routine use of these protected animals for research.
The development of an in vitro system for drug testing against this parasite has been impeded by the unavailability of the parasites in most laboratory settings, even though blood from infected humans has been used in prior screening of plant extracts [14]. Nevertheless, this source of parasites is ethically inappropriate. However, it has been shown that baboons inoculated with third-stage larvae of a human strain of $L$. loa developed patent infections after 135 to 148 days; in each animal which became patent, microfilaremia rose rapidly to high levels, followed by a suppression of microfilaremia during the 4th month of patency. After splenectomy, microfilariae reappeared in the peripheral blood in large numbers [13]. Thus, splenectomised baboons are a good animal reservoir of $L$. loa $\mathrm{mf}$. In this study, this source of parasite material was used to investigate the susceptibility of $\mathrm{mf}$ to L3 antiparasitic drugs.

The study described here addresses the in vitro activity of antiparasitic drugs on L. loa $\mathrm{mf}$ in culture. Ivermectin, an obvious control for this study, is the drug of choice for the treatment of onchocerciasis. This semi-synthetic macrolide is effective in vivo against $\mathrm{mf}$ (but not adult worms) of $L$. loa. It shows little in vitro activity against $\mathrm{mf}[15]$, and this is thought to be related to the requirement of the host immune system to mediate $\mathrm{mf}$ killing by ivermectin in vivo [16]. The anticancer imatinib (Gleevec; GLV) was used because it was recently added to the list of filaricidal drugs [17] and its microfilaricidal property was reported against Brugia [18]. The benzimidazole flubendazole (FLBZ) has been suggested as a candidate macrofilaricide for onchocerciasis [19], and has been extensively investigated in a range of animal models since its discovery over 40 years ago. The benzimidazoles are broad spectrum anthelmintics that bind preferentially to parasite $\beta$-tubulin leading to the disruption of microtubules and, ultimately, to the death of the parasite. FLBZ exhibits macrofilaricidal activity against Brugia pahangi [20, 21], Breinlia booliati [22], and, as a pro-drug of FLBZ (UMF-078), on Onchocerca ochengi [23]. In addition, several studies in other filarial species have indicated that FLBZ lacks or has only very little activity against $\mathrm{mf}$ stages. For example, it is ineffective against $B$. pahangi $\mathrm{mf}$ [21], and shows very low activity compared to ivermectin against $\mathrm{mf}$ in a mouse model of Onchocerca lienalis [24]. It was recently shown to inhibit the development of B. malayi mf to L3 in Aedes aegypti, while it did not affect the viability of $L$. loa and $B$. malayi $\mathrm{mf}$ at $10 \mu \mathrm{M}$ after $72 \mathrm{~h}$ exposure in vitro [25]. Praziquantel and the antimalarial drugs mefloquine, artesunate, chloroquine and amodiaquine were tested as they exhibit anthelmintic activity against Schistosoma species [26-30]. Fexinidazole and Scynexis-7158 are new clinical candidates currently under development against sleeping sickness. Since the endemicity of human African trypanosomiasis (HAT) overlaps with that of 
loiasis in some area of sub-Saharan Africa [1, 31], there is a need to predict the effect of these drugs on the viability of $L$. loa mf. This will help to prevent fatal complications such as those that have been observed post-mass treatment of onchocerciasis with ivermectin in loiasis co-endemic areas. These two new oral candidates were suggested by the Drug for Neglected Disease initiative (DNDi) and partners for clinical trial studies. Fexinidazole (5-nitro-imidazole), was successfully evaluated in Phase I between 2009 and 2012 and its potential as an oral, safe and well-tolerated drug was demonstrated [32, 33]. Phase II/III trials were launched in December 2012 in Democratic Republic of the Congo (DRC) followed by Central African Republic and was completed in November 2017. The oxaborole Scynexis7158 (SCYX-7158) entered the phase 1 clinical trials in March 2012 in Paris, and that was successfully completed by 2015 . These candidates were selected on the basis of their safety and their potential to treat advanced-stage sleeping sickness as observed in preclinical studies [34-36].

\section{Methods}

\section{Source of parasites}

Loa loa $\mathrm{mf}$ were obtained from baboons (Papio anubis) kept in captivity and infected with the human strain of L. loa according to a modified protocol of Orihel et al. $[12,13]$ and Wanji et al. [37]. Briefly, the animals were splenectomized and infected by subcutaneous injection of 100 infective larvae (L3) of L. loa from wild-caught Chrysops flies, with the animals being carefully monitored clinically and parasitologically until patency [38].

Baboons were acquired from hunters in different regions of Cameroon and maintained at a refurbished monkey house in Kumba. They were fed fruits, fats and proteins supplemented with vitamins.

\section{Culture constituents and test agents}

Complete culture medium (CCM) used to maintain the parasites in vitro was RPMI 1640 supplemented with $2 \mathrm{~g} / \mathrm{l}$ sodium bicarbonate, $25 \mathrm{mM}$ Hepes, $10 \%$ fetal bovine serum (FBS), $100 \mathrm{U}$ penicillin, $100 \mu \mathrm{g} / \mathrm{ml}$ streptomycin and $2.5 \mu \mathrm{g} / \mathrm{ml}$ amphotericin B. Stock-isotonic Percoll (SIP - Pharmacia, Uppsala, Sweden) was prepared by mixing 1 part 10x concentrated RPMI 1640 and 9 parts Percoll; dilutions were made in RPMI 1640. DMSO, RPMI 1640, Hepes, sodium bicarbonate, FBS and amphotericin B were purchased from Sigma Aldrich (St Louis, MO, USA) while penicillin-streptomycin solutions (Pen-Strep) were obtained from Gibco/Thermo Fisher (Grand Island, NY, USA). All chemicals were culture grade.

FLBZ and its principal metabolites, reduced flubendazole (R-FLBZ) and hydrolyzed flubendazole (H-FLBZ), were obtained from Epichem Pty Ltd, Murdoch University
Campus, South Street, Murdoch WA 6150, Australia. Imatinib was a gift from T. Nutman, NIH, Bethesda, MD, USA. Ivermectin (IVM), Praziquantel (PZQ), mefloquine (MFQ), artesunate (ATS), quinine (QN), chloroquine $(\mathrm{CQ})$ and amodiaquine (AQ) were purchased from Sigma-Aldrich (St Louis, MO, USA). Fexinidazole and SCYX-7158 were provided by DNDi.

\section{Preparation of parasites}

Blood was aseptically collected from infected baboons by venipuncture into EDTA vacuum tubes. Mf extraction began immediately upon arrival in the laboratory using a modified iso-osmotic Percoll extraction method $[39,40]$. Discontinuous gradient solutions containing 40\%, 50\% and $65 \%$ SIP were prepared in $15 \mathrm{ml}$ centrifuge tubes. An aliquot of $2 \mathrm{ml}$ of undiluted blood was pipetted and layered gently onto the gradient and the tubes centrifuged at $900 \times g$ for $10 \mathrm{~min}$ at $25^{\circ} \mathrm{C}$. The layer containing $\mathrm{mf}$ was removed with a syringe and filtered gently through a $5-\mu \mathrm{m}$ pore cellulose filter. The filter was immediately transferred to a Petri dish containing $\mathrm{CCM}$ and incubated at $37{ }^{\circ} \mathrm{C}$ for $5 \mathrm{~min}$. After removing the filter, the remaining fluid containing the $\mathrm{mf}$ was centrifuged $\left(300 \times g\right.$ for $10 \mathrm{~min}, 25{ }^{\circ} \mathrm{C}$ ), the $\mathrm{mf}$ quantified and transferred to culture plates. The quality of $\mathrm{mf}$ and the culture conditions were initially assessed by culturing the first batch of parasites for 40 days. During this pre-optimization step, $\mathrm{mf}$ retained optimal motility for at least 10 days, confirming that during the 5 days of screening, there would be minimal risk of $\mathrm{mf}$ dying due to the stress of the culture conditions. Immediately after purification, all batches were free of dead parasites, and the proportion of sluggish $\mathrm{mf}$ was usually below 1/100.

\section{Preparation of drug solutions}

Stock solutions of each drug were prepared by dissolving $5 \mathrm{mg}$ in $500 \mu \mathrm{l}$ DMSO in an Eppendorf tube which was then vortexed. For AQ, CQ and GLV, the DMSO component was replaced by double de-ionized water. Following this, $20 \mu \mathrm{l}$ of each suspension was transferred into $10 \mathrm{ml} \mathrm{CCM}$, then two-fold dilutions were made to obtain final concentrations between $0.156-10 \mu \mathrm{g} / \mathrm{ml}$. For the control, $20 \mu \mathrm{l}$ DMSO was mixed with $10 \mathrm{ml} \mathrm{CCM} \mathrm{to}$ make a $0.2 \%$ solution. The final concentration of DMSO was $0.1 \%$ in all wells including the negative controls.

\section{Cultures}

Parasites were cultured in 48-well plates. Each well contained $200 \mu \mathrm{l}$ drug solution at the desired concentration and $200 \mu \mathrm{l}$ parasite suspension containing $50 \pm 5 \mathrm{mf}$. For the control wells, $200 \mu \mathrm{l}$ DMSO (0.2\%) in CCM or CCM only were added to $200 \mu \mathrm{l}$ parasite suspension containing approximately $50 \mathrm{mf}$. Four replicates were tested for each drug concentration and each experiment was repeated 
twice. Motility was assessed under an inverted microscope at $24 \mathrm{~h}$ intervals for 5 days. The mf motility was scored on a scale of: 0 (immotile), 1 (intermittent shaking of the head and/or tail region), 2 (sluggish and motile), 3 (highly active and motile) [41]. On day 5, parasites with no movement were further assessed for viability by measuring the metabolic reduction of 3-(4, 5-dimethylthiazol-2-yl)-2, 5diphenyltetrazolium bromide (MTT) to formazan [42]. During this process, $\mathrm{mf}$ were incubated for $1 \mathrm{~h}$ in a 48 well plate containing $40 \mu \mathrm{l} /$ well MTT $(0.5 \mathrm{mg} / \mathrm{ml})$ in phosphate-buffered saline (PBS) and observed under an inverted microscope for color change in the parasite body [43]; live parasites stained blue while dead worms were unstained (Fig. 1).

\section{Data processing}

Cultures were evaluated on a relative scale. After scoring motility daily from day 0 of culture to the last day of each experiment by counting the number $(\mathrm{Ni})$ of parasites with the same score $(\mathrm{Si})$, motility (\%) of parasites in each well at each time point was summarized using the following formula:

$$
\operatorname{Motility}(\%)=\frac{\sum(\mathrm{N} i \times \mathrm{S} i)}{3 \times \sum \mathrm{S} i} \times 100
$$

Computed motility ranged from $0 \%$ if all $\mathrm{mf}$ in the well were immotile (score 0 ) to $100 \%$ when all worms are actively motile (score 3 , the maximum). This formula was built based on the evaluation of relative score

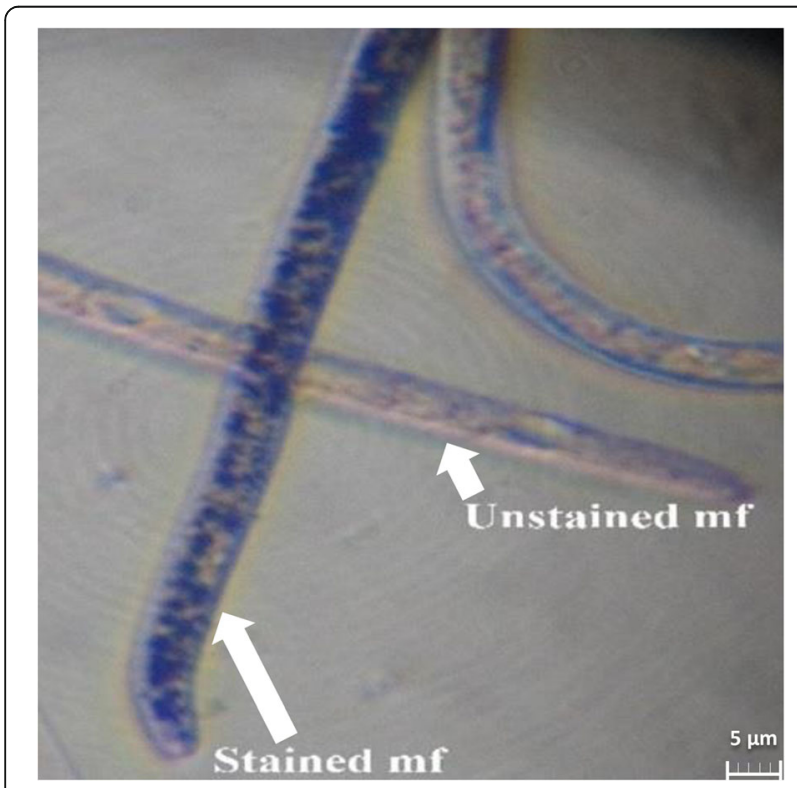

Fig. 1 Photomicrograph $(\times 400)$ of stained and unstained L. loa microfilariae after incubation with $\mathrm{MTT}$ reagent. This micrograph shows a live L. loa $\mathrm{mf}$ that metabolized MTT into blue formazan (left arrow) and a dead mf that is unstained (right arrow) described for an in vitro screening assay using albendazole, diethylcarbamazine and IVM against Brugia malayi infective larvae [44]. In the formula, the value 3 represents the highest score.

Percent reduction in motility for each drug concentration was calculated on days 3 and 5 using the following formula.

$$
\% \text { reduction }=\frac{\text { Control }- \text { drug }}{\text { Control }} \times 100
$$

The extent of mf mortality was reported as \% immotile (score 0) worms. Raw data were saved on a spreadsheet in Microsoft Excel 2013, and the motility response generated. Graphical displays were generated using GraphPad Prism software. Data were exported to SPSS version 20 for statistical analysis. Spearman's rank correlation rho was used to assess the association between the drug concentration and the reduction in mf motility on day 5 . The effect of drugs on the motility of parasites was also appreciated by estimating the concentration required for each drug to reduce the motility of the $\mathrm{mf}$ by $50 \%$, defined as $\mathrm{CR}_{50}$. The lower the value of $\mathrm{CR}_{50}$, the higher the activity of the drug. The $\mathrm{CR}_{50}$ was estimated using GraphPad Prism software. Results of replicates were expressed as mean \pm standard deviation (SD). The value of this parameter was used to compare the effects of the drugs on $\mathrm{mf}$. The effects of drug concentrations on motility were compared using non-parametric tests. The Kruskal-Wallis one-way analysis test was used to assess the global significant differences between the median $\mathrm{CR}_{50}$ of the active drugs and when a difference was detected, Dunn's post-hoc test was applied for pairwise multiple comparisons of the ranked data. This analysis was performed using the Pairwise Multiple Comparisons of Mean Rank Sums (PCMR) package in R version 3.1.4. Drugs were then grouped in ranked categories from the most active to the inactive. The distribution of median across drugs in the same rank was compared using either Mann-Whitney U-test or Kruskal-Wallis test. Statistical tests were interpreted using a 5\% significance level.

\section{Results}

Inhibitory effect of the anti-parasitic drugs on the kinetic of the motility of Loa loa mfs

A total of 13 anti-parasitic drugs were screened against $L$. loa $\mathrm{mf}$ (Additional file 1: Table S1). The susceptibility of parasite motility to the drugs over time is shown in Figs. 2 and 3. Generally, mf motility in negative control wells remained high and close to $100 \%$ over the 5 days of culture.

Figure 2 shows the $\mathrm{mf}$ motility with incubation time after treatment with filaricidal, anti-schistosomal and anticancer drugs. In the presence of FLBZ and its two metabolites at concentrations between $1.56-10 \mu \mathrm{g} / \mathrm{ml}, \mathrm{mf}$ motility decreased progressively with time. FLBZ (Fig. 2a) 


\section{a}

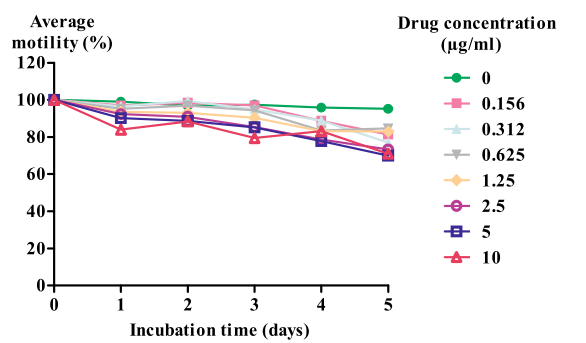

C

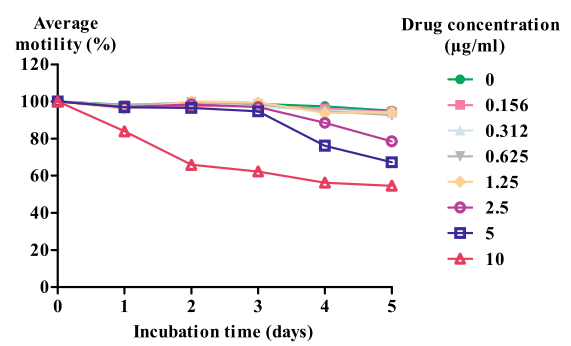

e

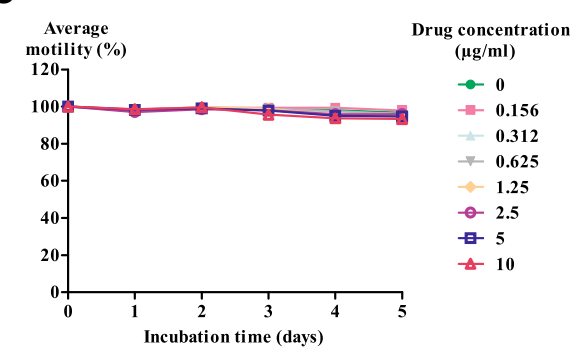

b

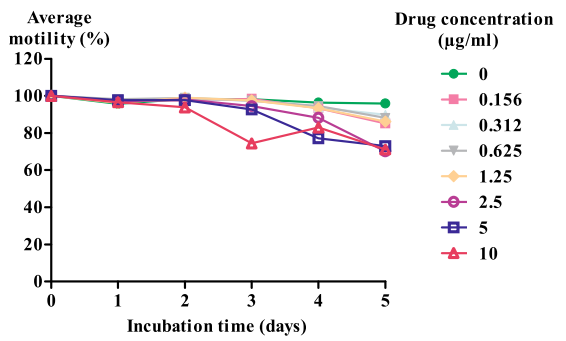

d

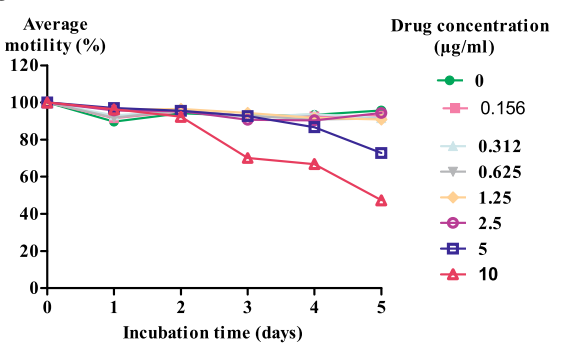

f

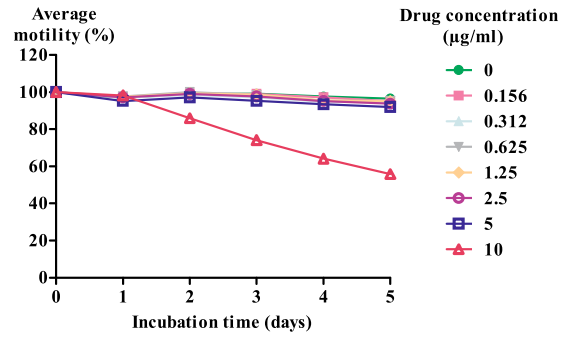

Fig. 2 Kinetics of the motility of L. loa microfilariae exposed to different concentrations of anti-filarial, anti-schistosomal and anti-cancer drugs within 5 days of incubation. a Flubendazole (FLBZ). b Reduced flubendazole (RFLBZ). c Hydrolysed flubendazole (HFLBZ). d Ivermectin (IVM). e Praziquantel (PZQ). f Imatinib (Gleevec, GLV)

and its reduced metabolite (Fig. 2b) displayed little activity against $L$. loa $\mathrm{mf}$ at concentrations $\leq 10 \mu \mathrm{g} / \mathrm{ml}$. The motility of $\mathrm{mf}$ decreased slightly from the first day after incubation and fluctuated around 70\% within the 5 days. Even at $10 \mu \mathrm{g} / \mathrm{ml}$, the motility of the parasites remained above $60 \%$. However, the hydrolysed metabolite affected mf motility from the first day at $10 \mu \mathrm{g} / \mathrm{ml}$ (Fig. 2c), and there was a decrease in worm motility with time. At 5 and $2.5 \mu \mathrm{g} /$ $\mathrm{ml}, \mathrm{mf}$ motility started to decrease from the third day, and the extent of reduction was concentration-dependent. At concentrations $<2.5 \mu \mathrm{g} / \mathrm{ml}$, mf motility was comparable to that of negative controls. Although reduction of $\mathrm{mf} \mathrm{mo-}$ tility was also observed from day 3 in the presence of 10 $\mu \mathrm{g} / \mathrm{ml}$ IVM to $47 \%$ on day 5 , the motility was still $>70 \%$ at concentrations $\leq 5 \mu \mathrm{g} / \mathrm{ml}$ (Fig. 2d). The antischistosomal PZQ had no effect on the motility of $L$. loa $\mathrm{mf}$ at $10 \mu \mathrm{g} / \mathrm{ml}$ (Fig. 2e). GLV reduced mf motility at $10 \mu \mathrm{g} / \mathrm{ml}$ from the first day of incubation to $55 \%$ by day 5 (Fig. 2f), and the reduction in motility was concentration-dependent.
The effects of antiprotozoal drugs on mf motility are illustrated in Fig. 3. The anti-malarial agents MFQ, AQ and CQ reduced the motility of $L$. loa $\mathrm{mf}$ after $48 \mathrm{~h}$ incubation at concentrations $>0.625 \mu \mathrm{g} / \mathrm{ml}$ (Fig. 3a, b and c, respectively). With AQ and MFQ, the reduction of $L$. loa mf motility was concentration-dependent, with MFQ being more active at higher concentrations. Reductions in mf motility were obtained with QN and ATS at 5 and $10 \mu \mathrm{g} / \mathrm{ml}$ (Fig. 3d and e, respectively); however; $\mathrm{mf}$ exposed to ATS showed no change in motility during the first $48 \mathrm{~h}$.

SCYX-7158 showed significant (Spearman's rho = -0.94285, $P=0.01667)$ concentration-dependent activity in reducing $L$. loa $\mathrm{mf}$ motility (Fig. 3f). At 12.5 $\mu \mathrm{g} / \mathrm{ml}$, which is close to the $C_{\max }$ values $(10 \mu \mathrm{g} / \mathrm{ml})$ found in mice after a dose of $25 \mathrm{mg} / \mathrm{kg}$, the reduction in motility was approximately $50 \%$ after 5 days of incubation. Fexinidazole had no significant effect on $\mathrm{mf}$ motility at $10 \mu \mathrm{g} / \mathrm{ml}$; all worms were actively motile (Fig. 3g). 

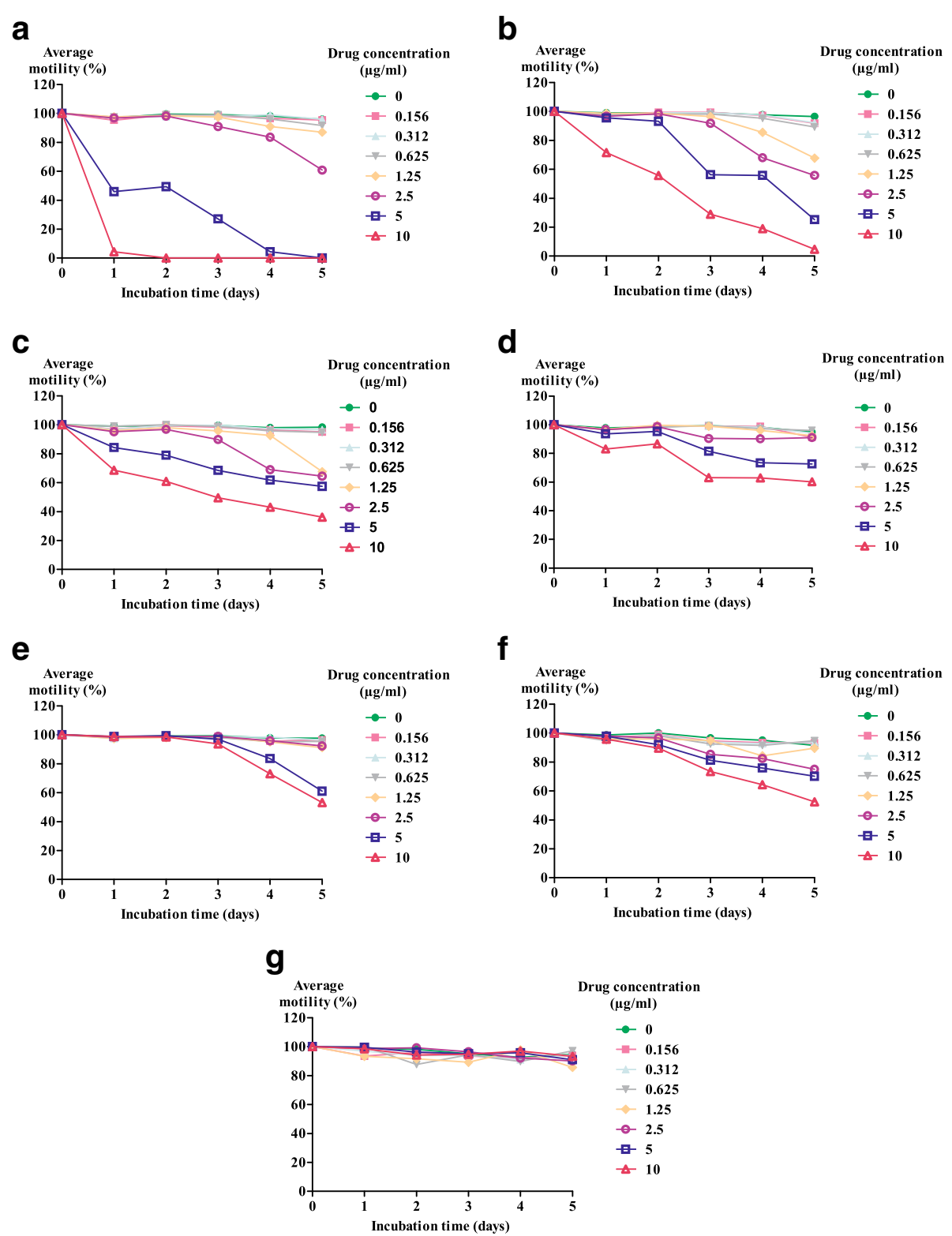

Fig. 3 Kinetic of the motility of L. loa microfilariae exposed to different concentrations of antiprotozoal drugs within 5 days of incubation. a Mefloquine (MFQ). b Amodiaquine (AM). c Chloroquine (CQ). d Quinine (QN). e Artesunate (ATS). f SCYX-7158. g Fexinidazole

\section{Relative reduction of $\mathrm{mf}$ motility and concentration reduction $50\left(\mathrm{CR}_{50}\right)$}

A summary of drug effects on mf motility of the mfs with respect to controls for the 3rd and 5th days in culture is shown in Table 1, and $\mathrm{CR}_{50}$ values are compared in Fig. 4. Drugs tested varied in their activity against $\mathrm{mf}$. The relative reduction of $\mathrm{mf}$ motility exposed to MFQ at $10 \mu \mathrm{g} / \mathrm{ml}$ was $100 \%$ by day 3 . The $\%$ reduction of mf motility after 5 days exposure to FLBZ, R-FLBZ and H-FLBZ at concentrations of $10 \mu \mathrm{g} / \mathrm{ml}$ was $25.3 \%, 25.8 \%$ and $42.8 \%$, respectively. Three days post-exposure of $\mathrm{mf}$ to these drugs at $10 \mu \mathrm{g} / \mathrm{ml}$ reduced $\mathrm{mf}$ motility by $18.5 \%, 24.2$ and $36.8 \%$, respectively.
The drugs that effected Loa $\mathrm{mf}$ in vitro most are MF and AMQ, while PZQ and fexinidazole displayed no activity. Based on $\mathrm{CR}_{50}$ values, the drugs tested were ranked in 5 categories in term of their effects (Table 1, Fig. 4). The first category includes the most active drugs with lowest $\mathrm{CR}_{50}$ values: MFQ and $\mathrm{AM}$, with $\mathrm{CR}_{50}$ values of $3.9 \pm 0.09$ and $4.0 \pm 0.06 \mu \mathrm{g} / \mathrm{ml}$, respectively. The second group, with $\mathrm{CR}_{50}$ values between 8 and $10 \mu \mathrm{g} / \mathrm{ml}$, includes $\mathrm{CQ}$, H-FBZ, IVM and ATS, while the third group $(12<$ mean $\mathrm{CR}_{50}<14 \mu \mathrm{g} / \mathrm{ml}$ ) (if $10 \mu \mathrm{g} / \mathrm{ml}$ was the highest concentration tested, you cannot have a $\mathrm{CR}_{50}$ value above this) was the group of QN and GLV. The fourth group included 
Table 1 Relative effects of the agents tested on mf motility at 3 and 5 days of culture at 1.25 and $10 \mu \mathrm{g} / \mathrm{ml}$ (\% reduction from controls) and the $\mathrm{CR}_{50}$ values after 5 days incubation

\begin{tabular}{|c|c|c|c|c|c|c|c|c|c|c|}
\hline \multirow[t]{2}{*}{ Ranking } & \multirow[t]{2}{*}{ Drug } & \multicolumn{2}{|c|}{$\begin{array}{l}\text { Motility reduction } \\
\text { after } 3 \text { days }\end{array}$} & \multicolumn{2}{|c|}{$\begin{array}{l}\text { Motility reduction } \\
\text { (\%) after } 5 \text { days }^{\mathrm{a}}\end{array}$} & \multicolumn{5}{|c|}{ Summary statistics for $\mathrm{CR}_{50}$} \\
\hline & & $1.25 \mu \mathrm{g} / \mathrm{ml}$ & $10 \mu \mathrm{g} / \mathrm{ml}$ & $1.25 \mu \mathrm{g} / \mathrm{ml}$ & $10 \mu \mathrm{g} / \mathrm{ml}$ & Mean \pm SD & Median & $\begin{array}{l}\text { Geometric } \\
\text { mean }\end{array}$ & $\begin{array}{l}\text { Interquartile } \\
\text { range }\end{array}$ & $95 \% \mathrm{Cl}$ \\
\hline \multirow[t]{2}{*}{1 (Highly effective) } & MFQ & 1.8 & 100 & 9.3 & 100 & $3.9 \pm 0.1$ & 3.8 & 3.9 & 0.16 & $3.7-4.0$ \\
\hline & AMQ & 2.7 & 70.6 & 29.8 & 95.1 & $4.1 \pm 0.1$ & 4 & 4.1 & 0.1 & $4.0-4.1$ \\
\hline \multirow[t]{4}{*}{2} & CQ & 3.7 & 10.3 & 31.3 & 63.3 & $8 \pm 0.2$ & 8 & 8 & 0.37 & $7.7-8.3$ \\
\hline & $\mathrm{H}-\mathrm{FLBZ}$ & 7 & 36.8 & 0.9 & 42.5 & $8.1 \pm 0.7$ & 8.1 & 8 & 1.36 & $6.9-9.3$ \\
\hline & IVM & 2 & 24.4 & 5 & 50.6 & $8.7 \pm 0.5$ & 8.6 & 8.7 & 0.83 & $8.1-9.3$ \\
\hline & ATS & 0.3 & 5.6 & 6.5 & 45.4 & $9.9 \pm 1.4$ & 10.1 & 9.8 & 2.58 & $7.7-12.1$ \\
\hline \multirow[t]{2}{*}{3} & QN & 0.4 & 36.4 & 2.7 & 36.5 & $12.7 \pm 0.9$ & 12.9 & 12.7 & 1.65 & $\begin{array}{l}11.8- \\
13.6\end{array}$ \\
\hline & GLV & 0.5 & 25.2 & 1.3 & 42.1 & $13.4 \pm 2.6$ & 12.8 & 13.2 & 4.64 & $9.3-17.5$ \\
\hline \multirow[t]{3}{*}{4} & R-FLBZ & 0.6 & 24.2 & 10.2 & 25.8 & $17.6 \pm 3$ & 17.8 & 17.5 & 5.56 & $\begin{array}{l}12.9- \\
22.4\end{array}$ \\
\hline & FLBZ & 7.2 & 18.5 & 12.9 & 25.3 & $21.2 \pm 3.2$ & 21.2 & 21 & 5.79 & $\begin{array}{l}16.1- \\
26.3\end{array}$ \\
\hline & SCYX-7851 & 0.1 & 18.1 & 1 & 31.4 & $25.4 \pm 5.6$ & 24.6 & 24.9 & 10.64 & $\begin{array}{l}20.3- \\
30.6\end{array}$ \\
\hline \multirow[t]{2}{*}{5 (Not effective) } & PZQ & 0.2 & 3.1 & 2.1 & 3.5 & - & - & - & - & - \\
\hline & Fexinidazole & 0.4 & 0.6 & 1.6 & 2.6 & - & - & - & - & - \\
\hline
\end{tabular}

Abbreviations: $\mathrm{Cl}$ confidence interval, $S D$ standard deviation, $-\mathrm{CR}_{50}$ values for of $\mathrm{PZQ}$ and fexinidazole were not estimated, because they were inactive against $L$. loa $\mathrm{mf}$

${ }^{\mathrm{a}} \%$ reduction relative to the negative control culture

drugs with very limited activity at $10 \mu \mathrm{g} / \mathrm{ml}$ (FLBZ and its reduced metabolite and SCYX-7851). The last group included the two inactive drugs, fexinidazole and PZQ, with undetermined $\mathrm{CR}_{50}$ values.

The Kruskal-Wallis chi-square value was $46.3636(d f=$ $10, P=1.232 \mathrm{e}-06)$, indicating significant differences in the activities between various active drugs. Within a group of drugs with similar activities, differences were determined using Mann-Whitney U-test and KruskalWallis test. Although MFQ and AQ were classified in the same group, MFQ had the significantly $(U=16, P=$ 0.02857 ) lowest value of $C_{50}$. However, no difference was found between the drugs within each of the remaining groups.

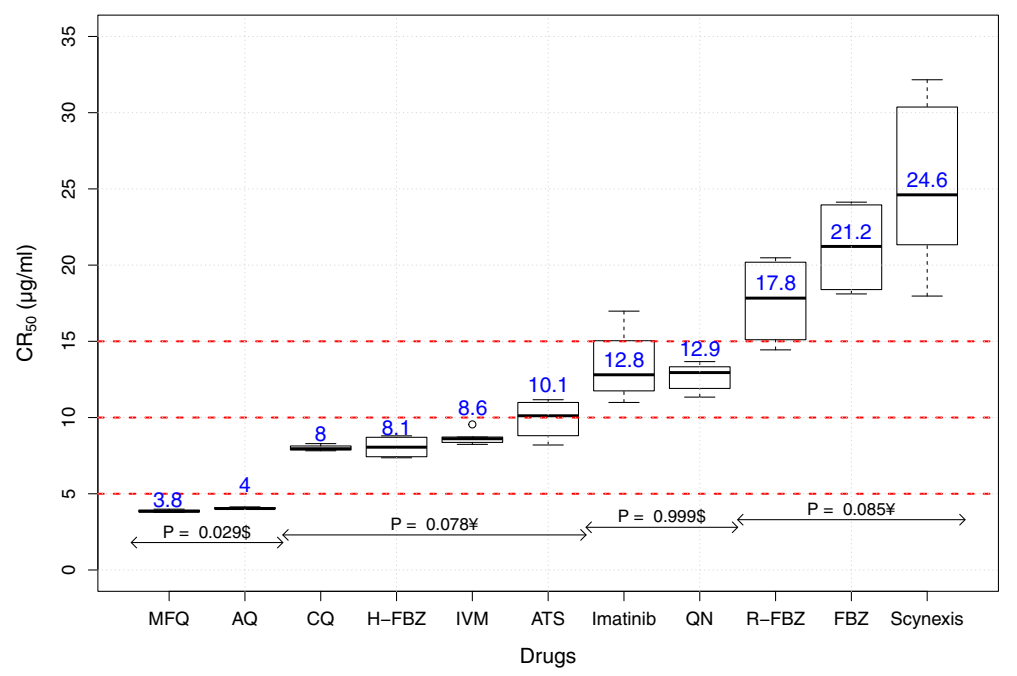

Fig. 4 Comparative activity of the various drugs against $L$. loa microfilariae. Box-plots show the activities of the various drugs ( $\left(R_{50}\right)$ against $L$. loa microfilariae. Fexinidazole and PZQ are not represented here because they were inactive. \$: $P$-value for Mann-Whitney U-test. $¥: P$-value for Kruskal-Wallis test 
Lethal effect of the anti-parasitic drugs on $\mathrm{mf}$

In addition to reduction in $\mathrm{mf}$ motility, lethal effects (score 0) were observed with some drugs (Fig. 5). This effect was more pronounced with MFQ than with AQ, given that at $10 \mu \mathrm{g} / \mathrm{ml}, 100 \%$ of $\mathrm{mf}$ were dead after $24 \mathrm{~h}$ (Fig. 5, Additional file 2: Table S2). Mortality rates for cultures exposed to MFQ increased with time and drug concentration. At $5 \mu \mathrm{g} / \mathrm{ml}$ MFQ the rates on day 1 and 5 were 4.5 and $100 \%$, respectively, while for $2.5 \mu \mathrm{g} / \mathrm{ml} \mathrm{AQ}$, these rates were 2.3 and $85.7 \%$, respectively. No immotile $\mathrm{mf}$ was found in wells containing FLBZ, H-FLBZ, fexinidazole, IVM or GLV at $10 \mu \mathrm{g} / \mathrm{ml}$, the highest concentration tested, at the 5 day time point. On day 4, SCYX-7158 showed lethal effect on $0.7 \%$ of the $L$. loa $\mathrm{mf}$ at $10 \mu \mathrm{g} / \mathrm{ml}$.

\section{Discussion}

Post-treatment SAEs constitute a significant challenge for mass drug administration programmes in countries with endemic onchocerciasis and lymphatic filariasis and co-endemic loiasis. IVM and diethylcarbamazine, the drugs of choice for these diseases, can induce SAE in Loa-infected patients $[4,45]$. As these SAEs are thought to be associated with the destruction of huge numbers of $\mathrm{mf}$ by these drugs, a principal aim of scientists and field program managers is to develop a way of reducing the circulating number of Loa $\mathrm{mf}$ without destroying a high number at once and thus avoid the SAEs. Development of such a drug requires investigation of the activity of selected candidates on $L$. loa $\mathrm{mf}$ using various experimental models. In this study, we assessed the predictive value of an in vitro system for the evaluation of drugs against loiasis.

FLBZ, a promising macrofilaricide candidate [19], was proposed to be useful in loiasis endemic areas based on the lack of activity against mf observed in various animal models [19]. Our results indicate that FLBZ at concentrations up to $10 \mu \mathrm{g} / \mathrm{ml}$ reduced the motility of $L$. loa $\mathrm{mf}$ progressively to a minor degree but did not kill the parasites during 5 days of culture. This indicates that the drug has relatively little direct effect on $L$. loa $\mathrm{mf}$ in vitro, and may not kill $L$. loa $\mathrm{mf}$ in vivo, as has been found with other filariae [21, 24]. The same finding was observed previously with $L$. loa and B. malayi mf exposed to FLBZ in vitro [25]. In this study, H-FLBZ was slightly more active against $\mathrm{mf}$ than FLBZ or R-FLBZ; however, it is generally thought that R-FLBZ but not $\mathrm{H}$ FLBZ is a bioactive anthelmintic, with the latter present at only very low plasma levels. Our findings support previous results with other filarial species that showed that FLBZ had little or no effect on $\mathrm{mf}[21,24]$. In earlier studies in humans, no evidence of immediate $\mathrm{mf}$ destruction was found after i.m. injections of FLBZ [46], which was supported by a notably slow reduction of the population of O. volvulus $\mathrm{mf}$ over time; likewise, there is a lack of microfilaricidal activity of this drug on $B$. pahangi [21]. FLBZ nevertheless appears to have significant effects on adult filariae, and in all likelihood considerably more than IVM does. Thus, its ability to eliminate adult worms $[20-23,47]$ without a potentially dangerous direct effect on $\mathrm{mf}$ makes it a good candidate

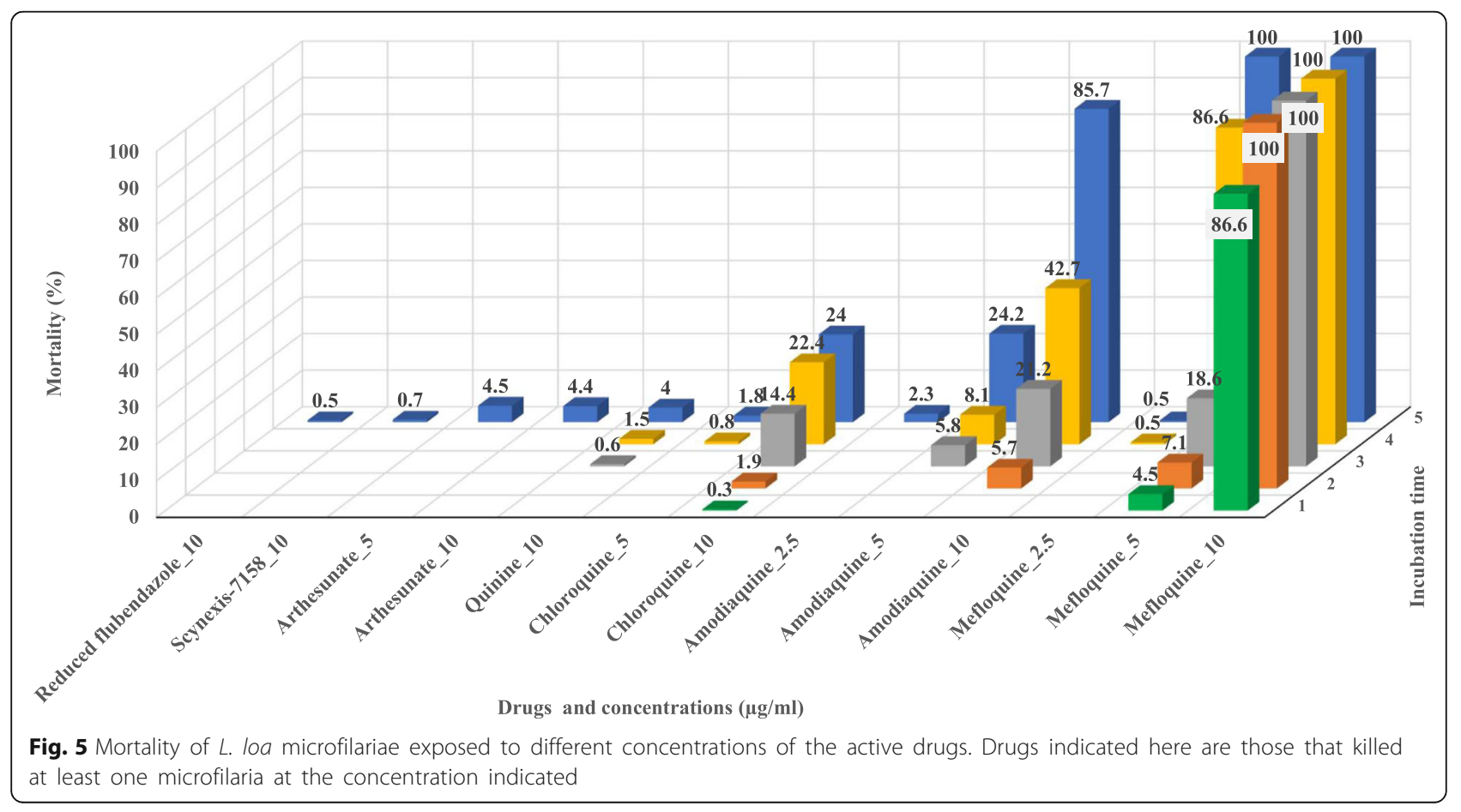


for use in the global elimination/eradication programs for filarial infections.

In vitro studies are useful in providing information concerning the direct effect of drugs against organisms such as filarial parasites. However, many antiparasitic drugs require a contribution from the host to be effective, components that are typically missing from in vitro assays; this indeed appears to be the case with IVM $[15,16]$. It is important, therefore, to compare in vitro results with results from in vivo models, and to include both positive and negative controls in any in vitro study. In this study, we included a range of chemotherapeutic agents that have previously been tested against filariae in vitro. Our findings are consistent in general with expectations from previous in vitro and in vivo work and indicate that our culture system over a period of five days produces comparable and valid data. Among the agents used for comparison here, the anti-malarials ATS, CQ, AQ and QN have all been found inactive against $L$. loa $\mathrm{mf}$ in humans [48]. Except CQ, whose in vivo microfilaricidal activity has been proven against $O$. volvulus [49], agents that are known to be active against filariae and that were also active in our present studies are MQ [25] and imatinib, an anti-cancer compound recently added to the list of active filaricidal drugs $[17,18,50]$. PZQ, an anti-schistosomal drug, was also tested but was inactive in this system.

HAT has considerable public health and economic impacts and is usually fatal when untreated. Current drugs suffer from poor safety profiles, inadequate regimens, limited effectiveness, and high cost. In an effort to develop alternative therapies, two new candidates, fexinidazole and SCYX-7158, were selected to enter preclinical studies on the basis of their safety and potential to treat advanced-stages of sleeping sickness. Most of the foci of sleeping sickness situated in the forest zones of East and Central Africa were identified as loiasis endemic areas, constituting an obstacle to onchocerciasis control and lymphatic filariasis elimination in sub-Saharan Africa due to SAEs resulting from massive killing of $L$. loa $\mathrm{mf}$ by IVM. It is important to determine if similar SAEs are as threat to the use of these new trypanocides.

Studies on $L$. loa-trypanosome co-infections have not been as extended as those on the co-occurrence of $L$. loa and Onchocerca spp. The heterogeneous nature of the associated clinical manifestations poses problems for prevention and timely patient management [51]. It is therefore obvious that any drug discovery strategy for HAT should take into consideration the issue of SAEs in co-infection with $L$. loa. To reduce a risk of SAEs, any drug to be massively used to control a disease in Loa endemic area, should have little or no effect on $L$. loa microfilaria.

Although fexinidazole and SCYX-7158 showed promising pharmacological activity and safety profiles in preclinical studies, their risk in inducing SAEs is not yet predicted. The present work thus evaluated fexinidazole and SCYX-7158 against L. loa mf in vitro. Fexinidazole had no significant effect on the viability of $L$. loa mf during 5 days of incubation. This highlighted the specificity of the drug to trypanosomes. In vitro trypanocidal activity of fexinidazole has already been investigated with $\mathrm{IC}_{50}$ range $(0.2-0.9 \mu \mathrm{g} / \mathrm{ml})$ against $T$. brucei species [35]. At a concentration close to 50 times these $\mathrm{IC}_{50}$ values, the drug had no lethal or paralyzing effect on $L$. loa $\mathrm{mf}$, suggesting that fexinidazole is compatible with MDA programme in Loa endemic areas. However, we cannot rely only on these data to predict the safety of fexinidazole in areas of loaisis endemicity, because it is known that even though IVM induced SAEs by killing massive numbers of $L$. loa $\mathrm{mf}$, it lacks direct microfilaricidal activity in vitro [15]. Therefore, it will be interesting to verify the specific activity of the drug in a murine model of loiasis and trypanosomiasis co-infection. Although the murine model of trypanosomiasis is already available, this is not a case with L. loa mf. However, there is an ongoing effort to develop a mouse model of loiasis.

SCYX-7158 exhibited little activity on $L$. loa mf. At concentrations between $12.5-50 \mu \mathrm{g} / \mathrm{ml}$, this drug inhibited the motility of $L$. loa $\mathrm{mf}$ by more than $50 \%$ by day 5 . In addition, on days 4 and 5 , close to $3 \%$ of $L$. loa $\mathrm{mf}$ were killed. However, these lethal concentrations were higher than plasma $C_{\max }$ levels $(10 \mu \mathrm{g} / \mathrm{ml})$ previously found in mice after a $25 \mathrm{mg} / \mathrm{kg}$ dose [36]. As discussed above, an in vivo system is required to validate the nonselectivity of this drug, and predict it risk of SAEs in loiasis co-endemic area.

\section{Conclusions}

This study reported, to our knowledge for the first time, the in vitro microfilaricidal activities of antimalarial drugs MFQ $\mathrm{AQ}, \mathrm{CQ}$, ATS and QN against L. loa mf. These findings highlight the need to investigate the effect of antimalarial therapy on loiasis microfilaraemia in the endemic areas. IVM, GLV, FLBZ and its major metabolites had little effect on $L$. loa $\mathrm{mf}$ in vitro. PZQ was inactive against $L$. loa $\mathrm{mf}$ in vitro. Fexinidazole and SCYX-7158 showed no and little effect, respectively, on $L$. loa $\mathrm{mf}$ viability at concentrations close to $C_{\max }$ values. However, further studies need to be done to confirm these findings in an in vivo model. These drugs remain candidates for clinical trials for sleeping sickness, even in areas of trypanosomiasis and loiasis coendemicity. Given that one cannot rely on in vitro activity to predict the effects of a drug because of pharmacokinetic and pharmacodynamic properties that are not incorporated in culture, it is advised to assess the effect of the above drugs in an in vivo model of loiasis. The selectivity of the drug on trypanosomiasis $v s$ loiasis can be assessed in an experimental model such as the recent development of patent infections of $L$. loa in a murine model. 


\section{Additional files}

Additional file 1: Table S1. Effects of different drug concentrations on the motility of L. loa $\mathrm{mf}$. (DOCX $33 \mathrm{~kb}$ )

Additional file 2: Table S2. Average rate of Loa mf mortality (\%) after exposure to different drug concentrations at different time intervals. (DOCX $20 \mathrm{~kb}$ )

\section{Abbreviations}

AQ: Amodiaquine; ATS: Artesunate; CCM: Complete culture medium; CQ: Chloroquine; $\mathrm{CR}_{50}$ : Concentration reduction 50; DMSO: Dimethyl sulfoxide; DNDi: Drug for Neglected Diseases initiative; FLBZ: Flubendazole; GLV: Gleevec (imatinib); H-FLBZ: Hydrolyzed flubendazole; $\mathrm{I}_{50}$ : Inhibitory concentration 50; IVM: Ivermectin; Mf: Microfilaria; MFQ: Mefloquine; MTT: 3(4,5-dimethylthiazol-2-yl)-2,5-diphenyltetrazolium bromide; PZQ: Praziquantel; QN: Quinine; R-FLBZ: Reduced flubendazole; SCYX-7158: Scynexis-7158

\section{Acknowledgements}

We sincerely thank Mr Bernard Synkalbe and Mr Bruno Oben for their technical support in taking care of baboons.

\section{Funding}

This work was funded through the financial support of the Bill and Melinda Gates Foundation through a grant provided to DNDi (Geneva).

\section{Availability of data and materials}

The data supporting the conclusions of this article are included within the article and its additional files. The raw datasets used and/or analyzed during the current study are available from the corresponding author upon reasonable request.

\section{Authors' contributions}

$A N, F F, M O, D Z, C N, P N$ and $A K$ carried out the parasite production, the culture work and/or the analysis of the data. SW, CM and TG developed the protocol and wrote the manuscript. All authors read and approved the final manuscript.

\section{Ethics approval and consent to participate}

Baboons (Papio anubis) used as parasite reservoir were handled according to international legislation and guidelines of the Cameroon National Veterinary Laboratory (LANVET, Ministry of Livestock, Fisheries and Animal Industry). The study design as well as different protocols was approved by the REFOTDE Institutional Animal Ethics Committee (RIAEC), with an ethical clearance obtained from this board, and the Cameroon National Ethics Committee (Ministry of Public Health). Handling of the animals and the investigations carried out were done strictly according to the international guidelines of rearing animals and using them in medical research under the official authorisation of the Ministry of Scientific Research in Cameroon (Research permit $\mathrm{N}^{\circ} 028 / \mathrm{MINRESI/B00/C00//C10/C12/2007).} \mathrm{The} \mathrm{manipulations} \mathrm{of} \mathrm{the}$ animals were done strictly according to the Animal Welfare Legislation and Policies, complied with the Animals (Scientific Procedures) Act 1986 (ASPA) and its associated codes of practice on animal housing and care [52]. Human participants were not involved in this study. Previous works that used the same procedures are [10,38].

\section{Consent for publication}

Not applicable.

\section{Competing interests}

The authors declare that they have no competing interests.

\section{Publisher's Note}

Springer Nature remains neutral with regard to jurisdictional claims in published maps and institutional affiliations.

\section{Author details}

${ }^{1}$ Parasites and Vectors Biology Research Unit (PAVBRU), Department of Microbiology and Parasitology, Faculty of Science, University of Buea, Buea, Cameroon. ${ }^{2}$ Institute of Parasitology, McGill University, Ste-Anne-de-Bellevue,
QC H9X 3V9, Canada. ${ }^{3}$ Biotechnology unit, Department of Biochemistry and Molecular Biology, Faculty of Science, University of Buea, Buea, Cameroon. ${ }^{4}$ Department of Biological Sciences, Western Michigan University, Kalamazoo, MI 49008, USA. ${ }^{5}$ Department of Pathobiology and Diagnostic Investigation, Michigan State University, East Lansing, MI 48824, USA. ${ }^{6}$ Filariasis

Programmes Support Unit, Liverpool School of Tropical Medicine, Pembroke Place, Liverpool L3 5QA, UK.

Received: 13 February 2018 Accepted: 15 March 2018

Published online: 04 April 2018

\section{References}

1. Zoure HG, Wanji S, Noma M, Amazigo UV, Diggle PJ, Tekle AH, Remme JH. The geographic distribution of Loa loa in Africa: results of large-scale implementation of the Rapid Assessment Procedure for Loiasis (RAPLOA). PLoS Negl Trop Dis. 2011;5:e1210.

2. Antinori S, Schifanella L, Million M, Galimberti L, Ferraris L, Mandia L, et al. Imported Loa loa filariasis: three cases and a review of cases reported in nonendemic countries in the past 25 years. Int J Infect Dis. 2012;16:e649-62.

3. Boulesteix G, Carme B. Encéphalite au cours du traitement de la filariose à Loa loa par la diéthylcarbamazine. A propos de 6 observations. Bull Soc Pathol Exot. 1986:79:649-54.

4. Gardon J, Gardon-Wendel N, Demanga N, Kamgno J, Chippaux J-P, Boussinesq $M$. Serious reactions after mass treatment of onchocerciasis with ivermectin in an area endemic for Loa loa infection. Lancet. 1997;350(9070):18-22.

5. Chippaux J-P, Boussinesq M, Gardon J, Gardon-Wendel N, Ernould J-C. Severe adverse reaction risks during mass treatment with ivermectin in loiasis-endemic areas. Parasitol Today. 1996;12:448-50.

6. Kamgno J, Boussinesq M, Labrousse F, Nkegoum B, Thylefors BI, Mackenzie $\mathrm{CD}$. Encephalopathy after ivermectin treatment in a patient infected with Loa loa and Plasmodium spp. Am J Trop Med Hyg. 2008;78:546-51.

7. VandeBerg JL, Williams-Blangero S, Tardif SD. The baboon in biomedical research. New York: Springer; 2009.

8. Wanji S, Amvongo-Adjia N, Koudou B, Njouendou AJ, Chounna Ndongmo PW, Kengne-Ouafo JA, et al. Cross-reactivity of filariais ICT cards in areas of contrasting endemicity of Loa loa and Mansonella perstans in Cameroon: Implications for shrinking of the lymphatic filariasis map in the Central African region. PLoS Negl Trop Dis. 2015;9:e0004184.

9. Bakajika DK, Nigo MM, Lotsima JP, Masikini GA, Fischer K, Lloyd MM, et al. Filarial antigenemia and Loa loa night blood microfilaremia in an area without bancroftian filariasis in the Democratic Republic of Congo. Am J Trop Med Hyg. 2014;91:1142-8.

10. Tendongfor N, Wanji S, Ngwa JC, Esum ME, Specht S, Enyong P, et al. The human parasite Loa loa in cytokine and cytokine receptor gene knock out BALB/c mice: survival, development and localization. Parasit Vectors. 2012;5: 43.

11. Azzibrouck GB, Akue JP, Lenoble DR. Production and immunological characterization of a recombinant subunit of a Loa loa polyprotein antigen. Parasitology. 2010;137:1119-28.

12. Orihel TC, Eberhard ML. Loa loa: development and course of patency in experimentally-infected primates. Trop Med Parasitol. 1985;36:215-24.

13. Orihel TC, Moore PJ. Loa loa: experimental infection in two species of African primates. Am J Trop Med Hyg. 1975;24:606-9.

14. Mengome LE, Akue JP, Souza A, Feuya Tchoua GR, Nsi Emvo E. In vitro activities of plant extracts on human Loa loa isolates and cytotoxicity for eukaryotic cells. Parasitol Res. 2010;107:643-50.

15. Devaney E, Howells RE. The microfilaricidal activity of ivermectin in vitro and in vivo. Trop Med Parasitol. 1984;35:47-9.

16. Moreno Y, Nabhan JF, Solomon J, Mackenzie CD, Geary TG. Ivermectin disrupts the function of the excretory-secretory apparatus in microfilariae of Brugia malayi. Proc Natl Acad Sci USA. 2010;107:20120-5.

17. Geary TG, Mackenzie CD. Adding "filaricide" to the Gleevec portfolio. J Infect Dis. 2015:212:677-80.

18. O'Connell EM, Bennuru S, Steel C, Dolan MA, Nutman TB. Targeting filarial Abl-like kinases: orally available, Food and Drug Administrationapproved tyrosine kinase inhibitors are microfilaricidal and macrofilaricidal. J Infect Dis, 2015:212:684-93.

19. Mackenzie CD, Geary TG. Flubendazole: a candidate macrofilaricide for lymphatic filariasis and onchocerciasis field programs. Expert Rev AntiInfect Ther. 2011;9:497-501. 
20. Van Kerckhoven I, Kumar V. Macrofilaricidal activity of oral flubendazole on Brugia pahangi. Trans R Soc Trop Med Hyg. 1988;82:890-1.

21. Denham DA, Samad R, Cho SY, Suswillo RR, Skippins SC. The anthelmintic effects of flubendazole on Brugia pahangi. Trans R Soc Trop Med Hyg. 1979;73:673-6.

22. Mak JW. Antifilarial activity of mebendazole and flubendazole on Breinlia booliati. Trans R Soc Trop Med Hyg. 1981;75:306-7.

23. Bronsvoort B, Makepeace B, Renz A, Tanya V, Fleckenstein L, Ekale D, Trees A. UMF-078: A modified flubendazole with potent macrofilaricidal activity against Onchocerca ochengi in African cattle. Parasit Vectors. 2008;1:18.

24. Townson S, Dobinson A, Connelly C, Muller R. Chemotherapy of Onchocerca lienalis microfilariae in mice: a model for the evaluation of novel compounds for the treatment of onchocerciasis. J Helminthol. 1988;62:181-94.

25. O'Neill M, Njouendou JA, Dzimianski M, Burkman E, Ndongmo PC, KengneOuafo JA, et al. Potential role for flubendazole in limiting filariasis transmission: observations of microfilarial sensitivity. Am J Trop Med Hyg. 2018;98:21-6.

26. Abou-Shady OM, Mohammed SS, Attia SS, Yusuf H-AS, Helmy DO. Therapeutic effect of mefloquine on Schistosoma mansoni in experimental infection in mice. J Parasit Dis. 2016:40:259-67.

27. Fahmy SR, Rabia I, Mansour EM. The potential role of mefloquine against Schistosoma mansoni infection by prohibition of hepatic oxidative stress in mice. J Basic Appl Zool. 2014;67:40-7.

28. Kato K, Miura M, Mitsui Y. In vitro effects of amodiaquine on paired Schistosoma mansoni adult worms at concentrations of less than $5 \mu \mathrm{g} / \mathrm{ml}$. Mem Inst Oswaldo Cruz. 2013;108:192-6.

29. Oliveira MF, d'Avila JC, Tempone AJ, Correâ Soares JB, Rumjanek FD, Ferreira-Pereira A, et al. Inhibition of heme aggregation by chloroquine reduces Schistosoma mansoni infection. J Infect Dis. 2004;190:843-52.

30. Keiser J, Silue KD, Adiossan LK, N'Guessan NA, Monsan N, Utzinger J, N'Goran EK. Praziquantel, mefloquine-praziquantel, and mefloquineartesunate-praziquantel against Schistosoma haematobium: a randomized, exploratory, open-label trial. PLoS Negl Trop Dis. 2014;8:e2975.

31. Simarro P, Cecchi G, Paone M, Franco J, Diarra A, Ruiz J, et al. The atlas of human African trypanosomiasis: a contribution to global mapping of neglected tropical diseases. Int J Health Geogr. 2010;9:57.

32. Torreele E, Trunz BB, Tweats D, Kaiser M, Brun R, Mazué G, et al. Fexinidazole - a new oral nitroimidazole drug candidate entering clinical development for the treatment of sleeping sickness. PLoS Negl Trop Dis. 2010;e923:4.

33. Tarral A, Blesson S, Mordt OV, Torreele E, Sassella D, Bray MA, et al. Determination of an optimal dosing regimen for fexinidazole, a novel oral drug for the treatment of human African trypanosomiasis: first-in-human studies. Clin Pharmacokinet. 2014;53:565-80.

34. Mäser P, Wittlin S, Rottmann M, Wenzler T, Kaiser M, Brun R. Antiparasitic agents: new drugs on the horizon. Curr Opin Pharmacol. 2012;12:562-6.

35. Kaiser M, Bray MA, Cal M, Bourdin Trunz B, Torreele E, Brun R. Antitrypanosomal activity of fexinidazole, a new oral nitroimidazole drug candidate for treatment of sleeping sickness. Antimicrob Agents Chemother. 2011;55:5602-8.

36. Jacobs RT, Nare B, Wring SA, Orr MD, Chen D, Sligar JM, et al. SCYX-7158, an orally-active benzoxaborole for the treatment of stage 2 human African trypanosomiasis. PLoS Negl Trop Dis. 2011;5:e1151.

37. Wanji S, Tendongfor N, Esum ME, Enyong P. Chrysops silacea biting densities and transmission potential in an endemic area of human loiasis in southwest Cameroon. Tropical Med Int Health. 2002;7:371-7.

38. Wanji S, Eyong EE, Tendongfor N, Ngwa C, Esuka E, Kengne-Ouafo A, et al. Parasitological, hematological and biochemical characteristics of a model of hyper-microfilariaemic loiasis (Loa loa) in the baboon (Papio anubis). PLoS Negl Trop Dis. 2015;9:e0004202.

39. Chandrashekar R, Rao UR, Rajasekariah GR, Subrahmanyam D. Isolation of microfilariae from blood on iso-osmotic percoll gradients. Indian J Med Res. 1984;79:497-501.

40. Van Hoegaerden M, Ivanoff B. A rapid, simple method for isolation of viable microfilariae. Am J Trop Med Hyg. 1986;35:148-51.

41. Njouendou AJ, Ritter M, Ndongmo WPC, Kien CA, Narcisse GTV, Fombad FF, et al. Successful long-term maintenance of Mansonella perstans in an in vitro culture system. Parasit Vectors. 2017;10:563.

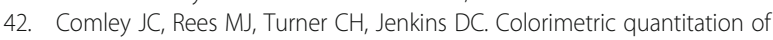
filarial viability. Int J Parasitol. 1989;19:77-83.

43. Comley JC, Townson S, Rees MJ, Dobinson A. The further application of MTT-formazan colorimetry to studies on filarial worm viability. Trop Med Parasitol. 1989;40:311-6.
44. Tippawangkosol P, Choochote W, Na-Bangchang K, Jitpakdi A, Pitasawat B, Riyong D. Comparative assessment of the in vitro sensitivity of Brugia malayi infective larvae to albendazole, diethylcarbamazine and ivermectin alone and in combination. Southeast Asian J Trop Med Public Health. 2004; 35:15-21.

45. Francis $H$, Awadzi $K$, Ottesen EA. The Mazzotti reaction following treatment of onchocerciasis with diethylcarbamazine: clinical severity as a function of infection intensity. Am J Trop Med Hyg. 1985;34:529-36.

46. Dominguez-Vazquez A, Taylor HR, Greene BM, Ruvalcaba-Macias AM, RivasAlcala AR, Murphy RP, Beltran-Hernandez F. Comparison of flubendazole and diethylcarbamazine in treatment of onchocerciasis. Lancet. 1983; 1(8317):139-43.

47. Zahner H, Schares G. Experimental chemotherapy of filariasis: comparative evaluation of the efficacy of filaricidal compounds in Mastomys coucha infected with Litomosoides carinii, Acanthocheilonema viteae, Brugia malayi and B. pahangi. Acta Trop. 1993;52:221-66.

48. Kamgno J, Djomo PN, Pion SD, Thylefors B, Boussinesq M. A controlled trial to assess the effect of quinine, chloroquine, amodiaquine, and artesunate on Loa loa microfilaremia. Am J Trop Med Hyg. 2010;82:379-85.

49. Guderian RH, Anselmi M, Beck BJ, Mackenzie CD, Williams JF, Proano JR, Cooper PJ. The effect of antimalarial chloroquine therapy and prophylaxis on concurrent infection with Onchocerca volvulus in Ecuador. Trans R Soc Trop Med Hyg. 1991;85:634-8.

50. Nutting CS, Eversole RR, Blair K, Specht S, Nutman TB, Klion AD, et al. Analysis of nematode motion using an improved light-scatter based system. PLoS Negl Trop Dis. 2015;9:e0003523.

51. Akue JP, Nkoghe D, Padilla C, Moussavou G, Moukana H, Mbou RA, et al. Epidemiology of concomitant infection due to Loa loa and Mansonella perstans in Gabon. PLoS Negl Trop Dis. 2011;5:e1329.

52. Hollands C. The animals (scientific procedures) Act 1986. Lancet. 1986; 328(8497):32-3.

\section{Submit your next manuscript to BioMed Central and we will help you at every step:}

- We accept pre-submission inquiries

- Our selector tool helps you to find the most relevant journal

- We provide round the clock customer support

- Convenient online submission

- Thorough peer review

- Inclusion in PubMed and all major indexing services

- Maximum visibility for your research

Submit your manuscript at www.biomedcentral.com/submit
) Biomed Central 